\title{
Practical Use of DNA Polymorphisms in the Avian Immunoglobulin Light Chain Constant Domain for Species-specific PCR
}

\author{
J. W. Choi *, S. J. Kang*, M. S. Park**, J.-K. Kim** and J. Y. Han*
}

Department of Agricultural Biotechnology, Seoul National University, Seoul, Korea*, Department of Microbiology, College of Natural Sciences, Changwon National University, Changwon, Korea**

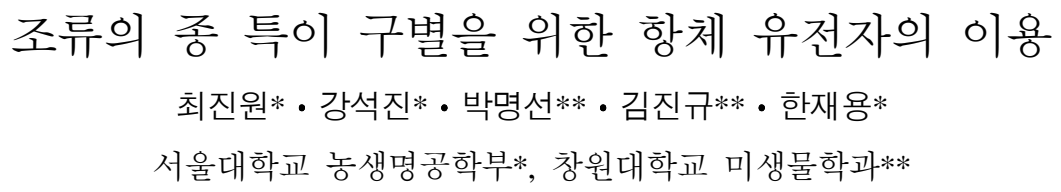

요 약

본 연구에서는 조류에서 종 특이적인 DNA 염기서열변이를 검증하기 위하여 닭, 낑, 칠면조, 메추 리의 immunoglobulin light chain constant domain 유전자를 클로닝하여 DNA염기서열을 분석하였다. 종 간에 구별이 가능한 DNA 염기서열변이가 위의 유전자에서 관찰되었다. PCR을 이용하여 종을 구별하 기 위하여 종 사이에 특이적인 DNA 염기서열 부위에 한 쌍의 종 특이적인 프라이머를 제작하였다. 또한 비교실험을 위하여 이미 알려진 cytochrome $b$ 와 tapasin 유전자에서도 두 쌍의 종 특이적 프라이 머를 제작하였다. PCR결과 세 쌍의 프라이머 모두 종 특이적으로 DNA를 증폭하였다. Immunoglobulin 유전자의 염기서열 변이를 이용한 종 특이적인 PCR 방법은 조류의 유전자원 보존을 위한 이종간 카 이메라 연구에 유용하게 이용될 수 있을 것이다.

(Key words : Avian inter-species chimera, Cytochrome b, Immunoglobulin light chain constant domain, Species-specific PCR, Tapasin)

\section{I . INTRODUCTION}

Species identification using accurate and reliable methods is necessary for the conservation of endangered species (Teletchea et al. 2005). In particular, the identification of avian species by molecular methods is important in studies of avian inter-species chimeras. The avian inter-species chimera, which has germ cells from different species, is a valuable model system for the conservation of endangered avian species.
DNA polymorphisms, which contain speciesspecific information, are used as markers for species identification. For example, the mitochondrial sequences encoding cytochrome $b$ (Jérôme et al., 2003 Wan et al., 2003) and 12S rRNA (Dalmasso et al. 2004 Rodríguez et al. 2003) are preferentially used because they display sufficient inter-specific variation and low intra-specific variation (Teletchea et al. 2005). Among the molecular methods used to detect species-specific DNA polymorphisms are PCR-

Corresponding author : Jae Y. Han, Department of Agricultural Biotechnology, Seoul National University, Seoul 151-921, Korea

Tel : +822-880-4810; Fax : +822-874-4811; E-mail : jaehan@snu.ac.kr 
RFLP (Hold et al. 2001), species-specific PCR (Rodríguez et al. 2003 Wan et al. 2003), and PCR-FINS (Jérôme et al. 2003). Of these techniques, species-specific PCR is the simplest because a single round of amplification is sufficient to detect species-specific DNA.

Species-specific markers can also be used to screen avian inter-species germline chimeras. Avian intra-species germline chimera are produced by transferring donor pluripotent cells to recipient embryos (Han et al. 2002; Kim et al. 2005; Park et al. 2003) resulting in more than two type of genetically different germ cells in their germline. Using the same method, avian inter-species germline chimeras are produced by transferring blastodermal cells (Li et al. 2002) or primordial germ cells (Ono et al. 1996) from different species. If the donor cells migrate to and proliferate in the gonads of the recipient embryo, they will produce donor-derived germ cells upon sexual maturation. Thus, using species-specific PCR, donor-derived DNA can be detected in sperm DNA samples. A similar strategy has been used to identify germline chimeric chickens using breed-specific PCR (Choi et al. 2007).

Here, we identified species-specific polymorphisms in the immunoglobulin light chain (IgL) gene among chicken, pheasant (Phasianus colchicus), quail (Coturnix japonica), and turkey (Meleagris gallopavo). Based on the differences in sequence between species, species-specific primers were designed for species-specific PCR. Additional species-specific primers were designed for the cytochrome $b$ and tapasin genes, for purposes of comparison with the IgL primers.

\section{ㅍ. MATERIALS AND METHODS}

\section{Animals}

White leghorn chickens, wild pheasants $(P$. colchicus), Japanese quail (C. japonica), and turkeys
(M. gallopavo) were used in this study. All procedures for animal management, reproduction, and surgery were performed in accordance with the standard operation protocols of Seoul National University, Seoul, Korea. Appropriate management of the experimental samples and quality control of the laboratory facility and equipment were also conducted. The animals were maintained at the University Animal Farm, College of Agriculture and Life Sciences, Seoul National University.

\section{Total RNA isolation and cDNA synthesis}

Total RNA was isolated from blood samples collected from the experimental animals using a PURESCRIPT $^{\circledR}$ RNA Purification System Blood Kit (Gentra Systems, Minneapolis, MN). cDNA was synthesized using Superscript III Reverse Transcriptase (Invitrogen, Carlsbad, CA).

\section{Cloning of the Immunoglobulin Light Chain Constant Domain}

$\mathrm{CKV}_{\mathrm{L}} \mathrm{B}$ and $\mathrm{CKV}_{\mathrm{L}} \mathrm{F}$ (Park et al. 2005), which are specific for the chicken immunoglobulin light chain variable domain (IgLV), were used to amplify immunoglobulin lighe chain constant domain (IgLC) from each of the above species. The products were then gel-extracted, ligated into pGEM-T Easy (Promega, Madison, WI), sequenced, and aligned using Clustal W 1.83 (Higgins et al. 1994).

3'-RACE PCR was used to clone the IgLC (Fig. 1). Based on the alignment, two gene-specific primers (Dieffenbach and Dveksler 2003), GSP1 (5'-CCTGGCAGTGCCCCTGTCAC-3') and GSP2 (5'- CACATTAACCATCACTGGGGTCC-3'), which match framework regions 2 and 3 in all species perfectly, were arbitrarily designed. Primer QT (5'-CCAGTGAGCAGAGTGACGAGGACTCGAGCTCAAGC TTTTTTTTTTTTTTTTT-3') was used for cDNA synthesis while primers Q0 (5'-CCAGTGAGCAGAGTGACG-3') and Q1 (5'-GAGGACTCGAGCT- 


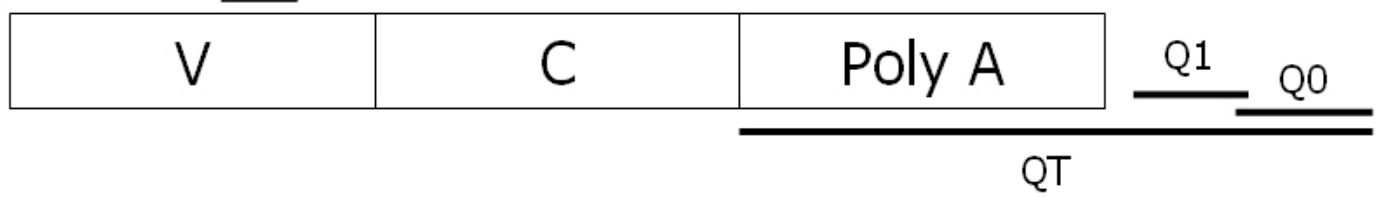

Fig. 1. Cloning of the immunoglobulin light chain by $3^{\prime}-\mathrm{RACE} P C R$. The mRNA sequence of avian immunoglobulin light chain $(\mathrm{IgL})$ is shown: variable domain $(\mathrm{V})$, constant domain (C), and poly A tail (Poly A). GSP1 and GSP2 are gene-specific primers (1 and 2) that anneal to framework 2 and 3, respectively. QT contains $17 \mathrm{Ts}$, in addition to overlapping sequences with Q0 and Q1 for primary and secondary nested PCR.

CAAGC- 3') were used for primary and secondary nested PCR. The secondary nested PCR products were gel-extracted and ligated into pGEM-T Easy vector (Promega).

\section{DNA Sequencing}

DNA sequencing was carried out using an $A B I$ Prism 3730 XL DNA Analyzer (Applied Biosystems, Foster City, CA). To minimize the possibility of error, three clones were sequenced per species and the highest quality clones, as judged from the chromatogram, were selected. The $I g L$ and cytochrome $b$ sequences from each species were aligned using ClustalW 1.83 (Higgins et al. 1994). The percent homology between each pair of species was calculated using Blast 2 Sequences (Tatusova and Madden. 1999).

\section{Species-specific PCR}

Genomic DNA was extracted from the blood of each species using a PUREGENE ${ }^{\circledR}$ DNA Purification Kit (Gentra Systems, Inc.) for use as the template. Based on sequence differences in the IgL (Fig. 2), cytochrome b (Fig. 3), and tapasin genes (Sironi et al. 2006), species-specific primers were designed (Table 2). All species-specific PCR was performed under the following conditions: $94^{\circ} \mathrm{C}$ for 3 min followed by 35 cycles of $94 \mathrm{C}$ for $30 \mathrm{~s}, 66^{\circ} \mathrm{C}$ (IgLC and cytochrome b), or $68^{\circ} \mathrm{C}$ (tapasin) for $30 \mathrm{~s}$, and $72 \mathrm{C}$ for $30 \mathrm{~s}$, plus a final extension at $72^{\circ} \mathrm{C}$ for 5 min.

\section{RESULTS AND DISCUSSION}

Primers specific for the chicken IgLV (Park et al. 2005) were successfully used to amplify DNA from chicken (control), pheasant, turkey, and quail species, and the amplified products were cloned and sequenced. The sequences were very similar in framework regions 2 and 3 (Fig. 2). To clone the unidentified constant domain, 3'-RACE-PCR was performed. Sequences from the variable domain to the poly A tail were cloned and sequenced in each species (Fig. 2). Cross amplification of the IgLV between species using the same primers indicates that both ends of the IgLV are similar among the members of the Phasianidae family, and it suggests that these primers may be used to clone the IgLV in other avian species.

Our alignment of the IgLC from each species indicates a high level of conservation; in fact, the level of homology between each pair of species was 83-95\% (Table 1). However, sufficient inter-specific sequence variation was identified to allow the development of species-specific primers, including a three-base-pair deletion in the pheasant, turkey, and quail $I g L$ gene at position 385 (Fig. 2), compared to the chicken IgL sequence (GenBank accession no. K00678.1). This indicates that the IgLC from any 
K00678. 1

White leghorn

Pheasant

Turkey

Quail

K00678. 1

White leghorn

Pheasant

Turkey

Quai I

K00678. 1

White leghorn

Pheasant

Turkey

Quai I

K00678. 1

Whi te leghorn

Pheasant

Turkey

Quai I

K00678. 1

Whi te leghorn

Pheasant

Turkey

Quail

K00678. 1

White leghorn

Pheasant

Turkey

Quail

K00678. 1

White leghorn

Pheasant

Turkey

Quail

K00678. 1

White leghorn

Pheasant

Turkey

Quai I
Signal peptide $\quad I \rightarrow$ Variable region

TCAGGTTCCCTGGTGCAGGCA |GCGCTGACTCAGCCGGCCTCGGTGTCAGCGAACCCGGGA 60 IGCGCTGACTCAGCCGTCCTCGGTGTCAGCAAACCTGGGA 39 - GCGCTGACTCAGCCGTCCTCGGTGTCAGCAAACCTGGGA 39 - GCGCTGACTCAGCCGTCCTCGGTGTCAGCAAACCCGGGA 39 | GGGCTGACTCAGCCGTCCTCGGTGTCAGCAAACCCAGGA 39 $* * * * * * * * * * * * * * * * * * * * * * * * * * * * * * * * * * * * * * * * * * * * * * *$ CDR1

GAAACCGTCAAGATCACCTGCTCCGGGGGT--------AGTGGCAGCTATGGC 105 GGAACCGTCGAGATCACCTGCTCCGGGGGTTACAGC---GACTATAGTGGCAACTATGGC 96 GAAACTGTCAAGATCACCTGCTCTGGGAGT---------AGCTATGCTTATGGC 84 GAAACCGTCAAGATCACCTGCTCCGGGGGT--------GCCTGGTATGGCTGGTATGGC 90 GAAACCGTCAAGGTCACCTGCTCCGGGGGTGGCAGCTATGCTGGAAGTTACTATTATGGC 99

$* * * * * * * * * * * * * * * * * * * * * * * *$

GSP1

CDR2

TGGTATCAGCAGAAGTCACCTGGCAGTGCCCCTGTCACTGTGATCTATAGCAACGACAAG 165 TGGTTCCAGCAGAAGTCTCCTGGCAGTGCCCCTGTCACTCTGATCTATAGCAACGACAAG 156 TGGTACCAGCAGAAGACACCTGGCAGTGCCCCTGTCACTGTGATCTATGGTAGCACTAGT 144 TGGTACCAGCAGAAGGCACCTGGCAGTGCCCCTGTCACTGTGATCTATGACAACACCAAC 150 TGGTTCCAGCAGAAGGCACCTGGCAGTGCCCCTGTCACTGTGATCTATGACAACAACAAC 159

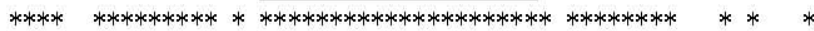

AGACCCTCGGACATCCCTTCACGATTCTCCGGTTCCAAATCCGGCTCCACAGCCACATTA 225 AGACCCTCGGACATCCCTTCACGATTCTCCGGTTCCAAATCCGGCTCCACAGCCACATTA 216 AGACCCTCGAACATCCCTTCACGATTCTCCGGTTCCAAATCCGGCTCCACAGCCACATTA 204 AGACCCTCGAACATCCCTTTACGATTCTCCGGTTCCAAATCTGGCTCCACACACACATTA 210 AGACCCTCGGACATCCCTTCACGATTCTCCGGTTCCCTATCCGGCTCCACAAACACATTA 219

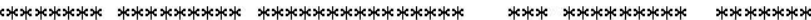

GSP2

ACCATCACTGGGGTCCAAGCCGAGGAGGAGGCTGTCTATTTCTGTGGGAGCTACGACAGC 285 ACCATCACTGGGGTCCAAGCCGAGGACGAGGCTGTCTATTTCTGTGGGAATGAAGACAGC 276 ACCATCACTGGGGTCCAAGCCGAGGACGAGGCTGTCTATTACTGTGGGAGCTACGACAGC 264 ACCATCACTGGGGTCCAAGCCGACGACGAGGCTGTCTATTACTGTGGGAATGCAGACAGC 270 ACCATCACTGGGGTCCAAGTCGAGGACGAGGCTATCTATTACTGTGGGAGTGCAGACAGC 279 $* * * * * * * * * * * * * * * * * * * * * * * * * * * * * * * * * * * * * * * * * * * * * * * * * * \quad * * * * * * * \pi$ J

$$
\text { CDR3 }
$$

---TATGTTGGTA ACCTATGTTGGTAGTTATGATGCTGCTATATTTGGGGCCGGGACAACCCTGACCGTCCTA 336 AGGATTATGGGTGGT---_-----ATATTTGGGGCCGGGACCACCCTGACCGTCCTA 312 AGTGGTGGTGGAA------TATTCGGGTCGGGGACCACCCTGACCGTCCTA 315 ACCAGTAGTGCTG-------------TATTCGGGTCCGGGACCATGTTGAACGTCCTA 323

$$
\text { * * }
$$$$
* * * * * * * * * * * * * * * * * * * * * * * * * * * *
$$

$$
I \rightarrow \text { Constant region }
$$

GGC I CAGCCCAAGGTGGCCCCCACCATCACCCTCTTCCCACCGTCAAAGGAGGAGCTGAAC 387 GGC| CAGCCCAAGGTGGCCCCCACCATCACCCTCTTCCCACCGTCAAAGGAGGAGCTGAAC 396 GGC| CAGCCCAAGGTGGCCCCCACCGTCACCATCTTCCCACCATCAAAGGAGGAGCTG--- 369 GGC| CAGCCCAAGGTGGCCCCCACCATCACCCTCTTCCCACCATCAAAGGAGGAGCTG--- 372 GGC | CAGCCCAAGGTGTCCCCCACCGTCAACCTCTTCCCACCATCAAAGGATGAGCTG--- 380

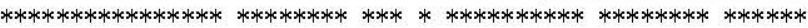

GAAGCCACCAAGGCCACCCTGGTGTGCCTGATAAACGACTTCTACCCCAGCCCAGTGACT 447 GAAGCCACCAAGGCCACCCTGGTGTGCCTGATAAACGACTTCTACCCCAGCCCAGTGACT 456 GAAAGCACCAAGGCCACCCTGGTGTGCCTGATAAACGACTTCTACCCCAGCCCGGTGACC 429 GATCAGAACAAGGCCACCCTAGTGTGCCTGATAAGCGACTTCTACCCCAGCCCGGTGACA 432 CAGCAGGGCAAGGCCACCCTGGTGTGCCTAATGAACGACTTCTACCCCAGCAAAGTGGAC 440 
$\begin{array}{ll}\text { K00678.1 } & \text { GTGGATTGGGTGATGGATGGCTCCACCCGCTCTGGCGAGACCACAGCACCACAGCGGCAG } 507 \\ \text { White leghorn } & \text { GTGGATTGGGTGATGGATGGCTCCACCCGCTCTGGGGAGACCACAGCACCACAGCGGCAG } 516 \\ \text { Pheasant } & \text { GTGGATTGGGTGATCGATGGCTCCACCCGCAGTGGGGAGAGCACAGCACGGCAGCGGCAG } 489 \\ \text { Turkey } & \text { GTGGAGTGGCTGGTGATGGCTCCACCCGGAAAGGGGAGACGACACCAGCGCAGCGGCAG } 492 \\ \text { Quail } & \text { GTGCAGTGGTCGGTCGATGGGACCACCTACAAAGGCGAGACCACAGCATCTCAGCGGCAG } 500\end{array}$

K00678.1 AGCAACAGCCAGTATATGGCCAGCAGCTATCTGTCACTGTCTGCCAGCGACTGGTCAAGC 567 Whi te leghorn AGCAACAGCCAGTATATGGCCAGCAGCTACCTATCACTGTCTGCCAGCGACTGGTCAAGC 576 Pheasant AGCAACAGCCAGTACATGGCCAGCAGCTACCTGTCACTGTCTGCCAGCGACTGGTCAAGC 549 Turkey AGCAACAGCCAGTACATGGCCAGCAGCTACCTGTCACTGACTGCCAGCGACTGGTCAAGT 552 Quail AGCAACAACCAGTACATGGCCAGCAGCTACCTGTCTCTGTCTGCCAACGACTGGGAAAGC 560 $* * * * * * * * * * * * * * * * * * * * * * * * * * * * * * * * * * * * * * * * * * * * * * * * * * * \quad * * *$

K00678. 1

CACGAGACCTACACCTGCAGGGTCACACACGACGGCACCTCTATCACGAAGACCCTGAAG 627 White leghorn CACGAGACCTACACCTGCAGGGTCACACACGACGGCACCTCTATCACGAAGACCCTGAAG 636 Pheasant CACGAGACCTACACCTGCAGGGTCACACACGACGGCACCTCTATCACGAAGACCCTGAaG 609 Turkey CACGAGACCTACACCTGCAGGGTCACACACGATGGCACCGCTGTCACGAAGACCCTGAAG 612 Quail CATGAGAACTACTCCTGCAGGGTCACACATGATGGCAACACTGTCACGAAGACCCTGAAG 620 $* * * * * * * * * * * * * * * * * * * * * * * * * * * * * * * * * * * * * * * * * * * * * * * * * * * * * * * * * * * *$ Stop

K00678. 1 AGGTCGGAGTGCTAATAGTCCCACTGGGGATGCAATGTGAGGACAGTGGTTCCTCACCCT 687 Whi te leghorn AGGTCCGAGTGCTAATAGTCCCACTGGGGATGCAATGTGAGGACAGTGGTTCCTCACCCT 696 Pheasant AGGTCGGAGTGCTCTTAGTCCCACTGGGGATGCGATGTGAGGACGGTGATTCCTCACCCT 669 Turkey AGGTCCGAGTGCTCT TAGTCCCACTGGGGATGCGATGTGAGGACGGTGGCTCCTCACCCT 672 Quail AGATCCGAGTGCTCT TAGACCCGCTGGGGGGGGATGTGAGGACGGTGGTTCCTCACCAT 680 $* * * * * * * * * * * * * * * * * * * * * * * * * * * * * * * * * * * * * * * * * * * * * * \quad * * * * * * * * * * *$

K00678. 1 CCCTGTCCCTCTGGGCCGCTGCTGGTGGCAGCAGCCCTCACTTCCCACTCAGATGTCCCC 747 Whi te leghorn CCCTGTCCCTCTGGGCGCTGCTGGTGGCAGCAGCCCTCACTTCCCACTCAGATGTCCCC 756 Pheasant CCCTGTCCCTCCGGGCCACTGCTGGTGACAGCAGCCCCCACTTCCCACTCAGATGTCTCC 729 Turkey CCCTGTCCCTCTGGGCCACTGCTGGTGGCAGCAGCCCCCACTTCCCACTCAGATGTCCCC 732 Quail CCCTGCTCCTCTGGTCAACTGCCGGTGGCAGCAGCCCCAACTTCCCCCTCAGATATCCCC 739 $* * * * * * \quad * * * * * * * * \quad * * * * * * * * * * * * * * * * * * * \quad * * * * * * * * * * * * * * * * * * * * * *$

K00678. 1 CACCGTGCCCCCATCACCCACCTCTGCCTGTTGACTCCTCTTGCCCTCATCTCTCCAGGT 807 Whi te leghorn CACCCTGCCCCCATCACCCACCTCTGCCTGTTGACTCCTCTTGCCCTCATCTCTCCAGGT 816 Pheasant CACCGTGCCCCCATCACCCACTTCTGCCTGTCGCCTCCTCTTGCCCCCATCCCTCCAGAT 789 Turkey CACCGTGCCCCCACCACCCACCTCTGCCTGTTGCCTCCTCTTGCCTCCATCCCTCCAGAT 792 Quail CACCATGCCCCCACCACCCACCTCTGCCTGTCGCCTCCTCTTGCCCCCACCCCTCCAGAT 799 $* * * * * * * * * * * * * * * * * * * * * * * * * * * * * * * * * * * * * * * * * * * * * * * \quad * * * * * * * * * * * *$

Poly A signal

K00678. 1 GTCACATTAATAAACACGACACTGAACTAGTGCTGACTCTGC 849 poly A

Whi te leghorn GTCACATTAATAAACACGACACTGAACTAGTGCTGACTCTGC 858 poly A

Pheasant GTCACATTAATAAACATGACACTGAACT---------- 817 poly A

Turkey GTCACATT AATAAACATGACACTGAACT-------- 820 poly A

Quail GTCACATTAATAAACATGACACTGAACTAGTGCTGACTCTGC 841 poly A

Fig. 2. Alignment of the immunoglobulin light chain $(\operatorname{Ig} L)$ sequences from white leghorn chicken, pheasant, turkey, and quail. The annealing sites for GSP1 and GSP2 are shaded. Nucleotides 366 and 478 of chicken IgL (K00678.1) (black shading) are the annealing sites of forward and reverse species-specific primers, respectively. The positions of the variable domain, the constant domain, the complementarity-determining regions (CDR), the $\mathrm{J}$ segment, and the poly $\mathrm{A}$ addition signal are taken from Reynaud et al. (1983). 
Table 1. Percent homology between the chicken, pheasant, turkey, and quail immunoglobulin light chain constant domain and cytochrome $b$ sequences

\begin{tabular}{ccccccc}
\hline \multirow{2}{*}{ Genes } & \multicolumn{7}{c}{ \% homology } \\
\cline { 2 - 7 } & $\begin{array}{c}\text { Chicken } \\
\text { /Pheasant }\end{array}$ & $\begin{array}{c}\text { Chicken } \\
\text { /Turkey }\end{array}$ & $\begin{array}{c}\text { Pheasant } \\
\text { /Quail }\end{array}$ & $\begin{array}{c}\text { Pheasant } \\
\text { /Turkey }\end{array}$ & $\begin{array}{c}\text { Pheasant } \\
\text { /Quail }\end{array}$ & $\begin{array}{c}\text { Turkey } \\
\text { /Quail }\end{array}$ \\
\hline \hline \multirow{2}{*}{ IgLC } & 95 & 90 & 83 & 92 & 85 & 86 \\
cytochrome $b$ & 86 & 86 & 86 & 86 & 86 & 85 \\
Tapasin & 73 & 76 & N/A & 92 & N/A & N/A \\
\hline
\end{tabular}

Blast 2 Sequences (Tatusova and Madden, 1999) was used to calculate the level of homology. The IgLC includes nucleotides 331-635 from K00678.1. The cytochrome $b$ sequence included the entire coding region, from the start codon to the stop codon. The tapasin sequence included exon 5 to 6 fragment of chicken (AJ004999.1), pheasant (AJ972781.1) and turkey (AJ972757.1) tapasin genes. The nucleotide sequence of quail tapasin is not available.

N/A : Not available.

species in which the IgLV is similar to that from chickens can be cloned using chicken IgLV-specific primers. In addition, direct amplification of an IgLC fragment using primers designed for the chicken IgLC is possible due to the high level of sequence similarity between species.

The potential for species identification based on inter-species polymorphisms in the IgLC was confirmed by PCR using species-specific primer sets (Table 2) which anneal to pheasant, turkey or quail but not chicken DNA due to a sequence difference in the IgLC. Additional sets of primer which amplify the cytochrome $b$ and tapasin genes were designed for comparison with the IgLC primers.

We used the cytochrome $b$ gene because it is a general vertebrate species-specific marker (Teletchea

Table 2. Pheasant-specific primers

\begin{tabular}{llc}
\hline Primer ID & \multicolumn{1}{c}{ Primer sequence (5'-3') } & Target gene \\
\hline \hline PSP-IgLC-F & ACCATCAAAGGAGGAGCTGGAA & \\
PSP-IgLC-R & GGTGCTGTGGTCTCGCCACT & \\
TSP-IgLC-F & ACCATCAAAGGAGGAGCTGGA TC & IgLC \\
TSP-IgLC-R & GCTGGTGTGGTCTCGCCTTT & \\
QSP-IgLC-F & ACCATCAAAGGATGAGCTGCAGC & \\
QSP-IgLC-R & TGAGATGCTGTGGTCTCGCCTTT & \\
PSP-cytob-F & CACACATGTCGAAATGTGCAG & \\
PSP-cytob-R & CTCATGGAAGGACATATCCTACG & \\
TSP-cytob-F & CTTGCATTCTCTTCTGTGGCCT \\
TSP-cytob-R & AGGACATAGCCTACAAAGGCTGTT \\
QSP-cytob-F & CCACACATGTCGAAACGTACAG & \\
QSP-cytob-R & GGTAAGACGTATCCTACGAAAGCA & \\
\hline PSP-tapasin-F & CAATGGTTAGGAGTGTCACAGGG & tapasin \\
TSP-tapasin-F & CCGTGGTTAGGAGGGATATTGA & \\
tapasin-R & GTAGAGCCAACGGATGAGGC & \\
\hline
\end{tabular}


Chi cken (AF354171. 1) Pheasant (AY368060. 1)

Turkey (L08381.1)

Quail (AF119094.1)

Chicken (AF354171.1) Pheasant ( $(Y 368060.1)$

Turkey (L08381.1)

Quail (AF119094.1)

Chi cken (AF354171. 1)

Pheasant (AY368060. 1)

Turkey (L08381. 1)

Quail (AF119094.1)

Chi cken (AF354171. 1)

Pheasant (AY368060. 1)

Turkey (L08381. 1)

Quail (AF119094.1)

TCCCTAGCCTTCTCCTCCGTAGCCCACACTTGCGGGAACGTACAATAGGGCTGACTCATC 240 TCCCTAGCCTTCTCTTCCGTAGCCCACACATGTCGAAATGTGCAGTACGGCTGACTCATC 240 ACTCTTGCATTCTCTTCTGTGGGCTACACATGCGGAAAGGTACAATACGGTTGACTCCTC 240 TCCCTAGCCTTCTCTTCCGTAGCGCACACATGTCGAAAGGTACAGT ACGGCTGACTCATT 240 $* * * * * * * * * * * * * * * * * * * * * * * * * * * * * * * * * * * * * * * * * * *$

CGGAATCTCCACGCAAACGGCGCCTCATTCTTCTTCATCTGTATCTTCCTTCACATCGGA 300 CGAAATCTCCATGCAAACGGCGCCTCATTCTTTTTCATTTGCATCTTCCTCCATATCGGA 300 CATAACCTCCATGCGAATGGGGCCTCATTCTTCTTCATCTGGATCTTCCTACACATTGGA 300 CGCAATCTCCATGCAAACGGGGCATCATTCTTCTTCATCTGCATCTTCCTCCACATCGGA 300 * *** ************************************************

CGAGGCCTATACTACGGCTCCTACCTCTACAAGGAAACCTGAAACACAGGAGTAATCCTC 360 CGCGGCCTCTATTACGGCTCTTACCTGTACAAAGAGACATGAAACACTGGAGTCGTCCTA 360 CGCGGCCTATATTATGGTTCGTACGTATATAAAGAAACGTGAAATAGAGGAGTAGTCTTA 360 CGAGGCCTATATTACGGCTCCTACCTITACAAAGAAACCTGAAACACAGGAGTAATCCTG 360

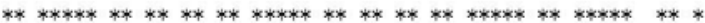

CTCCTCACACTCATAGCCACCGCCTTTGTGGGGTATGTTCTCCCATGGGGCCAAATATCA 420 CTCCTCACACTCATAGCAACCGCCTICGTAGGATATGTCGTTCGATGAGGACAAATATCA 420 CTTCTCACCCTCATAGCAAGAGCGTTTGTAGGCTATGTCGTTCCATGGGGACAAATATCA 420 CITCTCACACTAATAGCCACTGCTITCGTAGGATAGGTCTTACCATGAGGCCAAATATCC 420

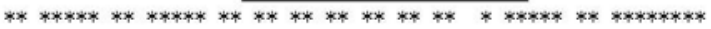

B

Chicken

Pheasant

CCGTGGTTAGGAGGGTCTGAGGGA

$-264$

Turkey

GAGTGGTTAGGTGGGTCTAAGGGACATTGAGATCATGGCTGGGGACACCAAGGGACACTG 300 CGGTGGTTAGG-----AGGGATATGA-TGATGGCTGGGGACACCAAGGGACACGG 290 $* * * * * * * * * *$ $* * * * * * *$

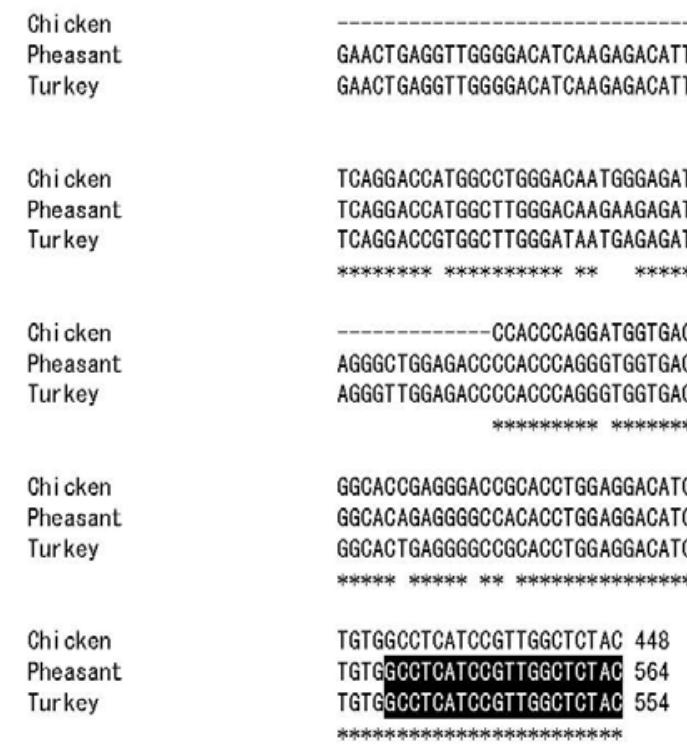

Fig. 3. Sequence alignment of the cytochrome $b$ (A) and tapasin (B) genes. (A) The chicken, pheasant, turkey, and quail cytochrome $b$ sequences were obtained from GenBank (accession numbers AF354171.1, AY368060.1, L08381.1, and AF119094.1, respectively). The annealing sites of the forward and reverse species-specific primers in cytochrome $b$ are shaded black. (B) The alignment result for tapasin is quoted from Shironi et al. (2006). The annealing sites of the species-specific primers in tapasin are shaded black. 


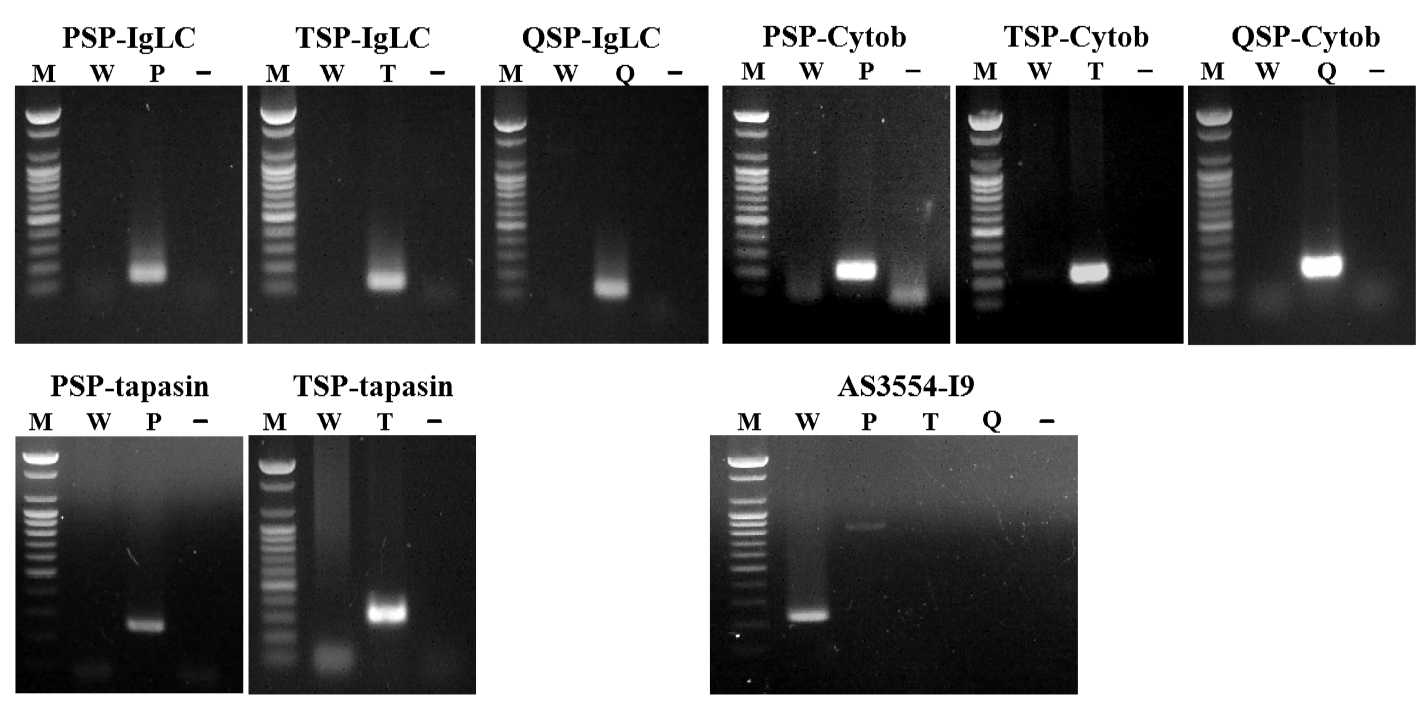

Fig. 4. Species-specific PCR using species-specific primers. The primer sets used are indicated at the top of each figure. M: 100-bp ladder, W: white leghorn genomic DNA, $\mathrm{P}$ : pheasant genomic DNA, T: turkey genomic DNA, Q: quail genomic DNA -: negative control (distilled water).

et al. 2005). The chicken, pheasant, turkey, and quail cytochrome $b$ sequences were obtained from GenBank (AF354171.1, AY368060.1, L08381.1, and AF119094.1) and aligned; for each pair of species, the level of homology was about $86 \%$ (Table 1). The species-specific primer sets in cytochrome $b$ were designed based on the regions with high inter-specific variations in cytochrome $b$ (Fig. 3A).

Tapasin was selected due to the existence of avian species-specific polymorphisms (Sironi et al. 2006). The primer set PSP-tapasin was designed on the basis of a sequence difference between pheasant and chicken involving a 95-bp insertion in pheasant tapasin, and the primer set TSP-tapasin which is specific for turkey was designed on the 8-bp deletion in turkey tapasin (Fig. 3B).

The species-specific primer sets produced products in their target species, whereas no amplification was detected in white leghorn (WL) chicken (Fig. 4). As a control, the primer set AS3554-I9 (Choi et al. 2007) was used to amplify a 222-bp fragment in WL chicken only (Fig. 4). Based on our results, our species-specific primer sets are species-specific and may be used to screen for interspecies germline chimeras between chicken and the other species including pheasant, turkey and quail.

The avian species-specific polymorphisms in the IgLC described in this study may be used as markers for species-specific PCR. This simple and unambiguous method may be applied to species identification and for screening avian inter-species germline chimeras.

\section{ACKNOWLEDGEMENTS}

This study was supported by a grant from the BioGreen 21 Program, Rural Development Administration, Republic of Korea, and by a graduate fellowship from the Brain Korea 21 Project of the Korean Ministry of Education.

\section{ABSTRACT}

Species-specific polymorphisms in chicken, pheasant, turkey, and quail were identified by cloning and sequencing of the immunoglobulin constant 
domain (IgLC). A set of species-specific primers were then designed on the basis of polymorphisms in the IgLC between species, as well as two additional sets of primers for the cytochrome $b$ and tapasin genes, for the purpose of species identification. Together, the primers successfully distinguished specific species from chicken by species-specific PCR. This simple but unambiguous method may be used to screen avian inter-species germline chimeras, which are valuable models for the conservation of endangered species.

\section{REFERENCES}

1. Choi, J. W., Lee, E. Y., Shin, J. H., Zheng, Y., Cho, B. W., Kim, J. K., Kim, H. and Han, J. Y. 2007. Identification of breed-specific DNA polymorphisms for a simple and unambiguous screening system in germline chimeric chickens. J. Exp. Zool. Part A Ecol. Genet. Physiol. 307: 241-248.

2. Dieffenbach, C. W. and Dveksler, G. S. 2003. PCR primer (ed.), 2nd ed., Cold Spring Harbor, New York, p 361.

3. Dalmasso, A., Fontanella, E., Piatti, P., Civera, T., Rosati, S. and Bottero, M. T. 2004. A multiplex PCR assay for the identification of animal species in feedstuffs. Mol. Cell. Probes. 18:81-87.

4. Han, J. Y., Park, T. S., Hong, Y. H., Jeong, D. K., Choi, K. D. and Lim, J. M. 2002. Production of germline chimeras by transfer of chicken gonadal primordial germ cells maintained in vitro for an extended period. Theriogenology. 58:15311539.

5. Higgins, D., Thompson, J., Gibson, T., Thompson, J. D., Higgins, D. G. and Gibson, T. J. 1994. CLUSTAL W: improving the sensitivity of progressive multiple sequence alignment through sequence weighting, position-specific gap penalties and weight matrix choice. Nucleic Acids Res. 22:4673-4680.

6. Hold, G. L., Russell, V. J., Pryde, S. E., Rehbein,
H., Quinteiro, J., Vidal, R., Rey-Mendez, M., Sotelo, C. G., Pérez-Martin, R. I., Santos, A. T. and Rosa, C. 2001. Development of a DNA-based method aimed at identifying the fish species present in food products. J. Agric. Food Chem. 49:1175-1179.

7. Jérôme, M., Lemaire, C., Verrez-Bagnis, V. and Etienne, M. 2003. Direct sequencing method for species identification of canned sardine and sardine-type products. J. Agric. Food Chem. 51: 7326-7332.

8. Kim, M. A., Park, T. S., Kim, J. N., Park, H. J., Lee, Y. M., Ono, T., Lim, J. M. and Han, J. Y. 2005. Production of quail (Coturnix japonica) germline chimeras by transfer of gonadal primordial germ cells into recipient embryos. Theriogenology. 63:774-82.

9. Li, Z. D., Deng, H., Liu, C. H., Song, Y. H., Sha, J., Wang, N. and Wei, H. 2002. Production of duck-chicken chimeras by transferring early blastodermal cells. Poult. Sci. 81:1360-1364.

10. Ono, T., Yokoi, R. and Aoyama, H. 1996. Transfer of male or female primordial germ cells of quail into chick embryonic gonads. Exp. Anim. 45:347-352.

11. Park, K. J., Park, D. W., Kim, C. H., Han, B. K., Park, T. S., Han, J. Y., Lillehoj, H. S. and Kim, J. K. 2005. Development and characterization of a recombinant chicken single-chain Fv antibody detecting Eimeria acervulina sporozoite antigen. Biotechnol. Lett. 27:289-295.

12. Park, T. S., Jeong, D. K., Kim, J. N., Song, G. H., Hong, Y. H., Lim, J. M. and Han, J. Y. 2003. Improved germline transmission in chicken chimeras produced by transplantation of gonadal primordial germ cells into recipient embryos. Biol. Reprod. 68:1657-1662.

13. Reynaud, C. A., Dahan, A. and Weill, J. C. 1983. Complete sequence of a chicken lambda light chain immunoglobulin derived from the nucleotide sequence of its mRNA. Proc. Natl. Acad. Sci. USA 80:4099-4103. 
14. Rodríguez, M. A., García, T., González, I., Asensio, L., Mayoral, B., López-Calleja, I., Hernández, P. E. and Martín, R. 2003. Identification of goose, mule duck, chicken, turkey, and swine in foie gras by species-specific polymerase chain reaction. J. Agric. Food. Chem.51:1524-1529.

15. Sironi, L., Lazzari, B., Ramelli, P., Gorni, C. and Mariani, P. 2006. Single nucleotide polymorphism discovery in the avian Tapasin gene. Poult. Sci. 85:606-612.

16. Tatusova, T. A. and Madden, T. L. 1999. Blast 2 sequences--a new tool for comparing protein and nucleotide sequences. FEMS Microbiol. Lett. 174:247-250.

17. Teletchea, F., Maudet, C. and Hänni, C. 2005. Food and forensic molecular identification: update and challenges. Trends. Biotechnol. 23:359-366.

18. Wan, Q. H. and Fang, S. G. 2003. Application of species-specific polymerase chain reaction in the forensic identification of tiger species. Forensic. Sci. Int. 131:75-78.

(접수일자 : 2007. 11. 15. / 채택일자 : 2008. 2. 12.) 